\title{
Functional characterization of the Ginkgo biloba chalcone synthase gene promoter in transgenic tobacco
}

\author{
L.L. Li ${ }^{1,2 *}$, H. Cheng ${ }^{1,2 *}$, H.H. Yuan ${ }^{1,2}$, F. Xu ${ }^{1}$, S.Y. Cheng ${ }^{1,2,3}$ and \\ F.L. $\mathrm{CaO}^{2}$
}

${ }^{1}$ Economic Forest Germplasm Improvement and Comprehensive Utilization of Resources of Hubei Key Laboratory, Huanggang Normal University, Huanggang, Hubei, China

${ }^{2}$ College of Forest Resources and Environment, Nanjing Forestry University, Nanjing, Jiangsu, China

${ }^{3}$ College of Chemistry and Life Science, Huanggang Normal University, Huanggang, Hubei, China

*These authors contributed equally to this study.

Corresponding author: C. Shuiyuan

E-mail: s_y_cheng@sina.com

Genet. Mol. Res. 13 (2): 3446-3460 (2014)

Received February 4, 2013

Accepted August 20, 2013

Published April 30, 2014

DOI http://dx.doi.org/10.4238/2014.April.30.6

\begin{abstract}
The regulative sequence (2273 bp) of the chalcone synthase gene promoter of biloba was cloned by genomic walking. A 2273-bp promoter 5' upstream translation start site of $G b C H S$ was cloned and designated as GbCHSP. pBI121+CHSP:GUS and pBI121-35S:GUS were constructed and transformed into tobacco by LBA4404. We found that GbCHSP could drive transient expression of GUS in tobacco and differentially expressed in root, stem and leaf tissues of this plant. GUS activity regulated by the CHSP promoter were located in tissues (apical meristems) at the growing points of roots and stems. pBI121+CHSP:GUS could be induced by wounding, copper, UV-B, abscisic acid, and ethephon treatments of transgenic seedlings. This activity was weakly
\end{abstract}


inhibited by gibberellin. Deletion analysis of the CHSP promoter in transgenic tobacco showed that $C H S P 1$ complete promoter conferred a GUS expression and activity similar to that of $35 \mathrm{~S}(\mathrm{CaMV})$. GUS activity dropped dramatically when there were $\mathrm{CHSP} 4, \mathrm{CHSP} 5$ constructs and was almost totally absent when the CHSP6 construct was present. We conclude that the upstream sequence -1548 to -306 of GbCHSP is the main region for transcriptional regulation of the $C H S$ gene and that it is activated by hormone and stress factors in G. biloba. These results will help us to understand the transcriptional regulatory mechanisms involved in $G b C H S$ expression and flavonoid accumulation in G. biloba.

Key words: Ginkgo biloba; CHSP; Transgenic tobacco; Functional analysis

\section{INTRODUCTION}

Ginkgo biloba is the only living representative of the older Ginkgoales, and it is also called a 'living fossil'. In the present time, G. biloba is one of the most popular functional plants, especially as a medicinal plant. Extracts of G. biloba leaves contain active compounds such as flavonoids and terpene lactones (ginkgolides and bilobalide) and can therefore be used to increase peripheral and cerebral blood flow (van Beek, 2002; Smith and Luo, 2004). Chalcone synthase (CHS), the first enzyme in the flavonoid biosynthetic pathway, is responsible for the establishment of the $\mathrm{C} 15$ skeleton of flavonoid compounds. Its activity is related to light intensity, and it is considered a key enzyme in regulating flavonoid biosynthesis (Heller and Hahlbrock, 1980; Xu et al., 2007a). Its transcription levels are increased by heredity, plant hormones, pathogenic attack, tissue wounding, UV irradiation, low temperature, or low levels of nitrogen and phosphate (Xu, 2005; Cheng et al., 2009). CHS is an inducible gene, and its promoter region has a number of cis-acting elements associated with adversity, hormones, tissue specificity, and other systems with inducible expression apart from key regulatory elements, such as ACE elements, silencers, H-box sequences, and AT-rich units (Weisshaar et al., 1991; Yu et al., 1993; da Costa e Silva et al., 1993; Kiba et al., 1995). The CHS gene promoter sequence of Arabidopsis thaliana contains the UV-A/UV-B/blue ray response element, and its expression is strongly induced by blue and ultraviolet rays (Hartmann et al., 1998). In Petunia hybrida and Pisum sativu, the presence of acting elements in the CHS promoter is correlated with the expression of adversity as well as temporal and spatial specificity (Schmid et al., 1990; van der Meer et al., 1992). Research into the CHS promoter in plants has shown that the transcriptional regulation of the CHS gene is realized through induced binding of MYB, bHLH, and other transcription factors (Koes et al., 2005). The expression of CHS genes is elaborately regulated by its growth as well as internal and external factors, as evidenced by the complex interaction between transcription factors and acting elements (Nakatsuka et al., 2003). Most studies of the CHS gene promoter have focused on its growth under adversity and the regulation of pigment synthesis, whereas research on the role of the CHS gene promoter in metabolite accumulation is limited (Liu et al., 2011). The interaction between the expression of regulatory elements upstream of the $C H S$ gene and trans-acting factors clearly merits further investigation as it will help regulate the accumulation of Ginkgo flavonoids. Studies on determining the role of the regulatory sequence of structural gene expression in the synthetic metabolism of Ginkgo 
flavonoids are crucial for identifying the molecular mechanisms involved in the entire process (Pang et al., 2005; Xu, 2005; Xu et al., 2007b). In this study, the upstream transcription regulatory sequence of the Ginkgo CHS gene was cloned via genome walking, and bioinformatics analysis was carried out. Moreover, as the objective of this study was to further examine the function of CHS regulatory elements and their relationship with encoded transcription factors, the CHS promoter was linked with the carrier containing the reporter gene GUS, and the function of CHS promoter in gene expression was demonstrated for the first time.

\section{MATERIAL AND METHODS}

\section{Plant material and treatments}

The different oligonucleotides used in this study are described in Table 1.

\begin{tabular}{|c|c|c|}
\hline Description & Serial number & Sequences $\left(5^{\prime}-3^{\prime}\right)$ \\
\hline \multirow[t]{8}{*}{ Genomewalker primers } & AP1 & GTAATACGACTCACTATAGGGC \\
\hline & AP2 & ACTATAGGGCACGCGTGGT \\
\hline & CP1 & TGAATGCCTCCAAGTCTTCCATCGCT \\
\hline & $\mathrm{CP} 2$ & AGTGGCTGGACCATCCGACCTCT \\
\hline & $\mathrm{CP} 3$ & GGAGGAGGCTATGGAAATTTAATGTCGT \\
\hline & $\mathrm{CP} 4$ & TTAGAAGGGTAGATAACTTGTAGCTC \\
\hline & CP5 & GCTTTGCCATCCGGAATTGTGTTAG \\
\hline & CP6 & GGTCTAATTCCTATCACTTTGGC \\
\hline \multirow{7}{*}{$\begin{array}{l}\text { Primers for CHSP1-6 amplification and } \\
\text { for construction of fusion vector }\end{array}$} & CHPD & TTggatccAGAAACTCGTTGTACGAACAAT \\
\hline & CHP1U & AAatcgatCTGGTAACTACATCTGCAGTTAT \\
\hline & CHP2U & AAatcgatTCCTGTCATCCCTACCTATTTC \\
\hline & CHP3U & AAatcgatCCAGAATAAGTAGGAGGTCAATGA \\
\hline & CHP4U & AAatcgatCCACATAATGAAGCACTACAAACTA \\
\hline & CHP5U & AAatcgatCGTAGCCTCTTTCAAAATGGT \\
\hline & CHP6U & AAatcgatTGAAATGCAGTCATAATTGCGC \\
\hline \multirow[t]{4}{*}{ Primers for verification } & CPU & CGAGTGCCAACGACCATGAAT \\
\hline & CPD & ACCCACACTTTGCCGTAATGAG \\
\hline & GUS1 & GTCAACGGGGAAACTCAGCAAG \\
\hline & GUS2 & TGAGCGTCGCAGAACATTACAT \\
\hline \multirow[t]{4}{*}{ Primers for QRT-PCR } & NIAU & TTCTTGAAAGATCACCCTGG \\
\hline & NIAD & CCAGGAGAGTCAGAGGTGTA \\
\hline & GUS3 & GGACTGTGACCTATACGGTG \\
\hline & GUS4 & TGGTAGAGATACGTGTTCAGTG \\
\hline
\end{tabular}

Twelve-year-old grafted G. biloba seedlings were grown in a greenhouse in Huanggang $\left(29^{\circ} 45^{\prime}-31^{\circ} 35^{\prime} \mathrm{N}, 114^{\circ} 54^{\prime}-116^{\circ} 8^{\prime} \mathrm{E}\right.$, Hubei Province, East China) were sampled as CHS promoter isolation materials. Tobacco plants (Nicotiana tabacum L. NC89) were grown under greenhouse conditions: 14 -h light at $24^{\circ} \mathrm{C}, 10$-h dark at $22^{\circ} \mathrm{C}$. Fortnight-old transgenic seedlings from the same genotypic strain of $N$. tabacum were subjected to treatments with UV-B, wounding (WOU), copper $\left(\mathrm{Cu}^{2+}\right)$, abscisic acid (ABA), gibberellin (GA), and ethephon (ETH). For UV-B treatment, seedlings were exposed to $1500 \mathrm{~J} / \mathrm{m}^{2} \mathrm{UV}-\mathrm{B}$ irradiation in a closed chamber, and the control cuttings were placed in a dark closed chamber. Copper treatment was performed by spraying $25 \mathrm{mM} \mathrm{CuSO}_{4}$ and control plants were sprayed with an equal amount of distilled water. The edges of tobacco leaves were cut by about $0.5 \mathrm{~cm}$ with scissors for wounding treatment, and the intact leaves of transgenic seedling were setting for control. 
$10 \mathrm{mM}$ ABA, $15 \mathrm{mM} \mathrm{GA}$, and $20 \mathrm{mM}$ ETH were dissolved in $0.01 \%$ Tween 20 and sprayed onto young leaves. The control leaves were sprayed with an equivalent volume of $0.01 \%(\mathrm{v} / \mathrm{v})$ Tween 20. All samples were immediately frozen in liquid nitrogen and stored at $-80^{\circ} \mathrm{C}$ prior to RNA extraction and GUS expression and activity analysis.

\section{DNA and RNA extraction}

Genomic DNA was extracted from the fresh leaves of $G$. biloba using the CTAB method described by Jiang and Cai (2000). To confirm transgene integration, total genomic DNA was isolated from the putative transgenic and control plants according to the method described by Porebski et al. (1997). Total RNA was extracted separately from all tobacco samples by using the Trizol Reagent kit (Invitrogen, USA) according to the manufacturer instructions, followed by incubation with RNase-free Dnase I (TaKaRa, Dalian, China) at $37^{\circ} \mathrm{C}$ for $30 \mathrm{~min}$. The quality and the concentration of genomic DNA and tobacco RNA were determined by agarose gel electrophoresis and spectrophotometer analysis.

\section{Isolation of the CHS promoter region}

The 5' genomic sequence flanking our cDNA sequence (Accession No. DQ054841) was cloned using the Universal Genome Walker kit (Clontech Laboratories, TaKaRa). Briefly, aliquots of high-molecular weight genomic DNA from Ginkgo were completely digested with different restriction enzymes that leave blunt ends. Each batch was ligated to the GenomeWalker adaptors and subjected to PCR amplifications. The primary round of PCR was carried out using the adaptor primer AP1 provided with the kit and a gene-specific primer $\mathrm{CP} 1$ in reverse orientation. Products were amplified by a second round of PCR using the nested set of primers AP2 and a gene-specific reverse primer CP2. On the basis of three times walker amplification, a fragment of approximately $2273 \mathrm{bp}$ was obtained from the $P v u \mathrm{II}$ library and inserted into the pM18-T vector (TaKaRa) for sequencing. This fragment comprising 2226 bp nucleotides upstream of the translation start of CHS was used in the present study.

\section{Construction of the GbCHS promoter: GUS plasmid and promoter deletion-GUS constructs}

A CHSP deletion-GUS construct was generated by fusing a CHS promoter fragment (from -2226 to $+1 \mathrm{bp}$, where the transcription start site was designated +1 (Figure 1) to the coding region of the GUS reporter gene in pBI121. Serially 5'-deleted CHSP1 to CHSP6 constructs were created by PCR using the reverse oligonucleotide primer CHPD above and six forward primers containing a ClaI restriction site at the 5'-ends: CHP1U, CHP2U, CHP3U, CHP4U, CHP5U, CHP6U. Each fragment was digested with ClaI/BamHI and subcloned into $\mathrm{ClaI} / \mathrm{BamHI}$-digested pBI121 to generate six promoter deletion derivatives. pBI121 were respectively digested with $\mathrm{ClaI}$ and $\mathrm{BamHI}$, the purity digested sequence and linear vector linked by T4 DNA ligase, then, GbCHSP1 to CHSP 6 were directly in frame cloned into the vector pBI121 (Figure 2). All constructs were verified by nucleotide sequencing. This vector contained the NPTII gene for kanamycin selection of putative transgenic plants. 


\begin{tabular}{|c|c|}
\hline 1 & 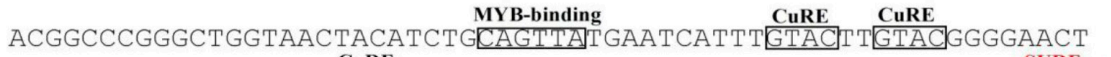 \\
\hline 61 & GGAACTGAAAGAGATATGTACAATGATCCGGGATATGAGATGCTCCTATTTATGG $\frac{\text { CURE }}{\text { SUATA }}$ \\
\hline 121 & TATTGCGAATGAATAGTCATCCTTCTAGATGATAGA \\
\hline 181 & $\begin{array}{l}\text { CuRE } \\
\text { TTTGATTIGTACATAGTCTATTTACAATACCGA }\end{array}$ \\
\hline 241 & AAACGAAATTCGAATCATAT \\
\hline 301 & GGIAC \\
\hline 361 & $\begin{array}{l}\text { GTATTTCCTTCCCTATAGAAGAts } \\
\text { CGAAATAATAGAGAAGAACGATAGA }\end{array}$ \\
\hline 421 & TTCGAATAGAGCATCAACTCTTAGTTATTGAATAATACCACATGCP \\
\hline 481 & CGAGAGACCTGTTTCTATTTCTCTTTGAATCTTTTTCTAAAAAAGC \\
\hline 541 & TCGGTAGAACGTAGGTCTCCAAAACCTGATGTCGTAGGTTCAAATCCTACAGAGCGTGAT \\
\hline 601 & TCTGTTCCTATTGGATCAAATTGGATTGATGGATCATCAATAACTCCAACTGGAACGATT \\
\hline 661 & $\begin{array}{l}\text { GAAGGATGTTCCTCAATCA } \\
\text { binding }\end{array}$ \\
\hline 721 & JTGATCACCACGTCGGTATTGTAAATACGL \\
\hline 781 & AGTGATAGGAATTAGAC \\
\hline 841 & CGGGATGAGTAAG \\
\hline 901 & TTCAG Ca-cis \\
\hline 961 & GTCACAAATTAACATAATG \\
\hline 1021 & СAACPABTAACATCACTACA \\
\hline 1081 & CGAAGAGCTAGAGCTAACACATGTCATAAGCAAAACGTAACTCGAGACCACCC \\
\hline 1141 & $\begin{array}{l}\text { CAGTCC-box } \\
\text { GAGTCCCGTCCIICTAGAAAACTACAACATGGAACCCTCGC }\end{array}$ \\
\hline 1201 & 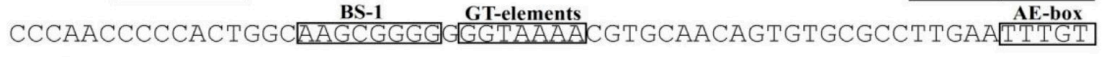 \\
\hline 1261 & TTCTACAATGTTAAATCTCCATAGCCTCCTCCAAAATGGTCAACGTGAAGTAACATTGTG \\
\hline 1321 & TAATTTCAAT \\
\hline 1381 & CTACCATTCTAATATCGGTGTTGTTGGGTGTGTGCATGTGCAAATTACAAATTATIGTCT \\
\hline 1441 & CTGAAAGTTGTTAATAATAAATCGGATGCGCCTATGGTGTGCACCTTAGAATTATTTTTA \\
\hline 1501 & AAATGGTCAACGT \\
\hline 156 & CACATCAA AGAATATCTATGTTCATCTCCGAGAGCTACA. \\
\hline 1621 & TGTTGTTAGATGCATGTGTATGCAAATTACAAACTAETGTTTCTAAA \\
\hline 1681 & TAATADATAAATCGGATACACCTACAATGTAAACCT \\
\hline 1741 & ПTTCCATAGCCTCСTCСАAАATGGTTAATGTAAAGTAACATTGTATAAT \\
\hline 1801 & GACCCOACGTCAAAAGAAATCTACGCTTATCTCGGAGAGTTACAAGGTATCTGTCATTCC \\
\hline 1861 & ATTGATGTTGTTGGACGCATGTGTGTACAAATTACAAATTATTATCTCTACCCGTCG \\
\hline 1921 & ATTGTTTLIS \\
\hline 1981 & $\begin{array}{l}\text { GC-motif } \\
\text { CCCCGTGGCCTCCAAAATGATCATCGTAAT } \\
\end{array}$ \\
\hline 2041 & CCATATCAAAGGATATCTCCGTTCATCTGGGAT, \\
\hline 2101 & TCAGCTTTGAACA GTCGCCAGCACGTGCCTTCAATCTAACCTCGCGAGTGCCAACGACCA \\
\hline 2161 & GTGGCGAATGAGTATAAAACTTGGCAGCGGCAGAGACCCCATATGTTCTC \\
\hline 2221 & 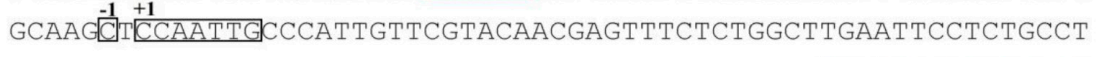 \\
\hline 2281 & CCGAGAGCATTCATCGCAGTATTGCATAGTACGACCGCAAGAGCGATGGAAGACTTGGAG \\
\hline & GCACAGAGGTCGGATGGTCCAGCCACTA \\
\hline
\end{tabular}

Figure 1. Nucleotide sequence (part) and in silico prediction of TBFs of the GbCHS promoter region. 


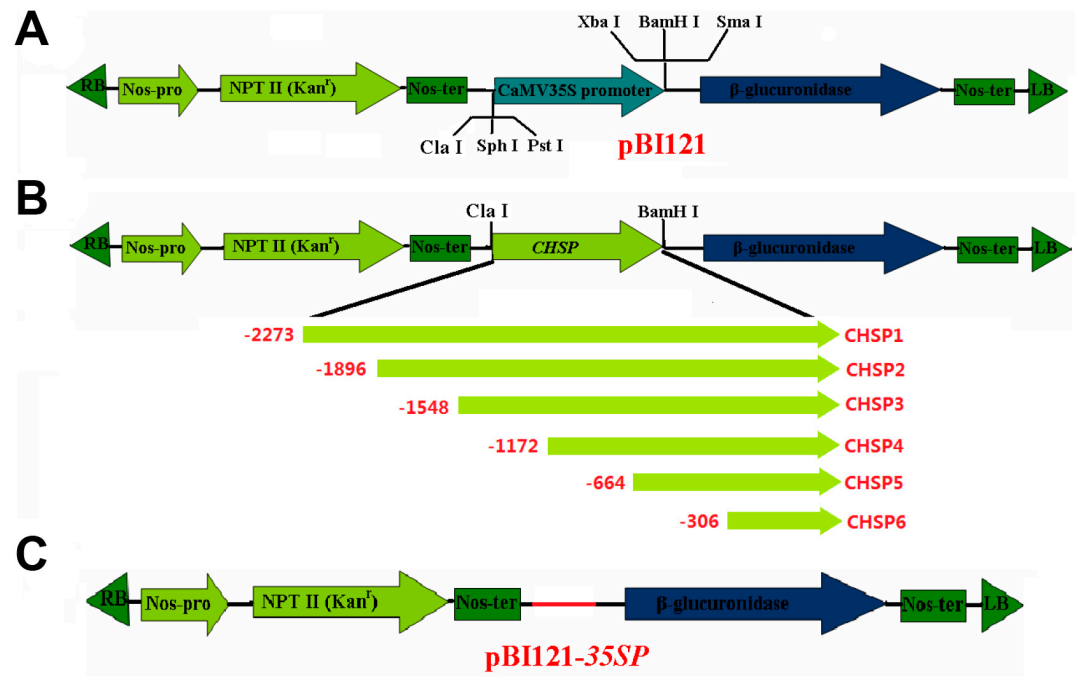

Figure 2. Construction of expression vectors. A. pBI121 vector take as the positive control. B. Full-length sequences of CHSP (CHSP1, CHSP2, CHSP3, CHSP4, CHSP5, CHSP6) were linked to pBI121, with the CaMV $35 \mathrm{~S}$ promoter removed using PCR to obtain $\mathrm{pBI} 121+\mathrm{CHSP} 1 \sim \mathrm{CHSP}$. C. $\mathrm{pBI} 121$ without the $35 \mathrm{~S}$ promoter were taken as the negative control.

\section{Transformation of vectors into Agrobacterium tumefaciens LBA4404}

A. tumefaciens strain LBA4404 transformed with pBI121-(CHSP1 6) construct was used to transform tobacco leaf discs as described by Li et al. (2010), and the main process was as follows: $1 \mu \mathrm{g}$ pBI121-(CHSP1 P6) plasmid was added to $200 \mu \mathrm{L}$ LBA4404 competent cells, which had been treated with $20 \mathrm{mM} \mathrm{CaCl}_{2}$ two times beforehand, and sterile glycerin was added to a final concentration of $25 \%$, followed by gentle mixing. The mixture was ice bathed for $30 \mathrm{~min}$, and then immersed in liquid nitrogen for $1 \mathrm{~min}$ before incubating at $37^{\circ} \mathrm{C}$ for $5 \mathrm{~min}$. Next, $1 \mathrm{~mL}$ YEP liquid culture medium without antibiotic was added to the mixture, which was agitated gently at $28^{\circ} \mathrm{C}$ for 3 to $4 \mathrm{~h}$. Next, $100 \mu \mathrm{L}$ of this new mixture were laid on the YEP plate with antibiotic ( $50 \mu \mathrm{g} / \mathrm{mL}$ kanamycin and $40 \mu \mathrm{g} / \mathrm{mL}$ rifamycin). The plate inversion was incubated at $28^{\circ} \mathrm{C}$ for $48 \mathrm{~h}$.

Leaves of aseptic tobacco seedlings were cut into segments of about $0.5 \sim 0.8 \times 0.5 \sim 0.8$ $\mathrm{cm}$ and dipped in the suspension of $A$. tumefaciens LBA4404 with pBI121-(CHSP1-P6) $(\mathrm{OD} 600 \mathrm{~nm}=0.5)$ for 6 9 min, and at the same time, some of the explants were treated with LBA4404 with only pBI121 vector as the negative control. The segments were then cultured on MS medium containing $0.5 \mathrm{mg} / \mathrm{L} 6-\mathrm{BA}$ and $0.1 \mathrm{mg} / \mathrm{L} \mathrm{NAA}$ at $28^{\circ} \mathrm{C}$ in the dark for 2 days before they were transferred onto the selection MS medium containing $0.5 \mathrm{mg} / \mathrm{L}$ 6-BA, 0.1 $\mathrm{mg} / \mathrm{L} \mathrm{NAA}, 500 \mathrm{mg} / \mathrm{L}$ carbenicillin and $100 \mathrm{mg} / \mathrm{L}$ kanamycin for shoot induction at $24^{\circ} \pm 2{ }^{\circ} \mathrm{C}$ under 2000 lx. When the kanamycin-resistant shoots were about $1 \sim 2 \mathrm{~cm}$ in height, they were transferred to the rooting medium, which was MS medium containing $0.1 \mathrm{mg} / \mathrm{L}$ NAA, 500 $\mathrm{mg} / \mathrm{L}$ carbenicillin and $80 \mathrm{mg} / \mathrm{L}$ kanamycin for rooting at $24^{\circ} \pm 2^{\circ} \mathrm{C}$ under $2000 \mathrm{~lx}$. When the roots were about $2 \mathrm{~cm}$, the plants were transferred to a greenhouse. The transgenic plants were grown under standard conditions. 


\section{Expression pattern of CHSP1 6 promoter analysis}

Three single-copy plants were chosen from each of six transgenic tobacco strains (CHSP1 CHSP6), and the transcription level and enzymatic activity of the GUS were subsequently measured. Real-time PCR was performed, and the tobacco gene nitrate reductase (Nia gene, GenBank accession code X14059) was taken as the internal reference against which to measure the transcription level and number of copies of the GUS reporter gene (AF354046) in tobacco plants. The NIAU, NIAD, GUS3 and GUS4 primers were designed on the basis of the sequences deposited at GenBank and quantitative real-time PCR (QRT-PCR) requirements.

The gene-specific primers (GUS3, GUS4) and reference primers (NIAU, NIAD) for QRT-PCR are listed in Table 1. The QRT-PCR conditions were: $10 \mathrm{~min}$ at $95^{\circ} \mathrm{C}$ and 40 cycles of $95^{\circ} \mathrm{C}$ for $15 \mathrm{~s}$ and $60^{\circ} \mathrm{C}$ for $1 \mathrm{~min}$ ). Before performing QRT-PCR, primer efficiency was evaluated using both Nia gene and GUS at 100, 150, 200, 250 and $300 \mathrm{nM}$ combinations. A $150 \mathrm{nM}$ concentration was chosen as the most suitable combination for both genes.

For each plant sample, aliquots of $150 \mathrm{ng}$ total RNA were analyzed for each gene and the two genes (Nia and GUS) were always analyzed simultaneously. Each sample was amplified 3 times and all reactions were performed on an ABI PRISM 7500 Sequence Detection System, and all data were analyzed by variance analysis with SPSS 16.0. With the housekeeping gene $N$. tabacum Nia, the relative amount of the GUS transcript was presented as $2^{\text {(-dCt) }}$ according to the $\mathrm{C}_{\mathrm{T}}$ method $\left(\mathrm{dCt}=\mathrm{Ct}_{\text {sample }}-\mathrm{Ct}_{\text {control }}\right)$ described in the QRT-PCR Application Guide (Applied Biosystems). When comparing the expression of GUS in different treatment and construction, the relative expression of GUS was achieved by calibrating its transcription level relative to that of the reference gene, $N$. tabacum Nia.

\section{GUS activity assays}

GUS activity was monitored using either histochemical or fluorometric assays according to Jefferson et al. (1987). Histochemical analyses were performed on plantlets from primary transformants propagated in vitro. For fluorometric assays, normalization of samples was performed by protein quantification (Hirsinger et al., 1999). Relative GUS activities were calculated and expressed as pmol MUG per minute per milligram protein. Three replicates were performed for each sample.

\section{Statistical analysis}

The experimental results are reported as means \pm standard error (SE) of three replicates. The data were analyzed using Statistical Product and Service Solutions (SPSS, Version 16.0) at $\mathrm{P}<0.05$ (Fisher's protected least significant difference).

\section{RESULTS}

\section{Identification and characteristics of a flavonoid-specific promoter $\mathrm{CHSP}$}

A 2.3-kb fragment harboring the -2273 to +25 region (translation initiation site of CHS as +1 ) upstream of CHS was isolated from Ginkgo leaves (Figure 1). Sequence analysis showed that this fragment contained several basal regulatory elements (Table 2), including seven TATA boxes 
for RNA polymerase binding and thirteen CAAT boxes for transcription frequency regulation (Figure 1). The GbCHSP promoter also contained several environmental factors, the cell cycle inductive response elements and cis-acting elements associated with hormone induction (Table 2).

Table 2. Cis-acting element analysis of promoter sequences from Ginkgo biloba CHS by PlantCARE.

\begin{tabular}{|c|c|c|c|c|c|c|c|}
\hline \multirow[t]{2}{*}{ Element name } & \multicolumn{6}{|c|}{ Promoters of GbCHS } & \multirow[t]{2}{*}{ Annotation } \\
\hline & 1 & 2 & 3 & 4 & 5 & 6 & \\
\hline CAAT-box & -2146 & -2023 & -1590 & -1424 & -518 & -96 & Common cis-acting element in promoter and enhancer regions \\
\hline CCAAT-box & -1954 & -1502 & -1349 & -369 & -133 & & To increase the heat shock genes promoter activity \\
\hline TATA-box3 & -1817 & -1312 & -880 & -878 & -439 & -44 & Common cis-acting element in promoter and enhancer regions \\
\hline TATA-box 5 & -736 & & & & & & Common cis-acting element in promoter and enhancer regions \\
\hline Inr-element & -1693 & -1396 & & & & & Light-responsive transcription of psaDb depends on Inr \\
\hline GT-element & -2107 & -1947 & -1002 & & & & Light-regulated \\
\hline Sure & -2111 & & & & & & Regulated by sucrose \\
\hline GATA-motif & -2154 & -2001 & -1827 & -528 & -257 & -176 & Part of a light responsive element \\
\hline $\mathrm{CBF}$ & -1324 & & & & & & Dehydration-response \\
\hline $\mathrm{Ca}-\mathrm{Cis}$ & -1332 & -997 & -106 & & & & Ca-cis element \\
\hline ABRE-like & -1525 & -1113 & -1033 & -690 & -421 & -105 & Etiolation and dehydration-response \\
\hline BIHD & -1251 & -1126 & -376 & & & & Defense and stress responsiveness \\
\hline Antiox & -1036 & & & & & & Antioxy response \\
\hline CuRE & -2185 & -1389 & -1093 & -495 & -340 & -261 & Copper-response element \\
\hline STK-1 & -1296 & -452 & -221 & -216 & & & $\mathrm{~K}$ channel regulation \\
\hline CCGTCC-box & -1081 & & & & & & Meristem specific activation element \\
\hline BS-1 & -1010 & & & & & & Transcription associated with vascular tissue \\
\hline MYB & -2201 & -1505 & & & & & MYB binding site \\
\hline MYC & -927 & & & & & & MYC binding site \\
\hline CIR & -235 & & & & & & Cis-acting regulatory element involved in circadian control \\
\hline EEC & -391 & +24 & +39 & & & & Enhancer elements \\
\hline Q-Element & -1573 & & & & & & Enhancer elements \\
\hline ARF & -792 & & & & & & Auxin-responsive element \\
\hline TGA-box & -1036 & & & & & & Auxin-inducement \\
\hline ABRE & -582 & -347 & & & & & Abscisic acid responsiveness \\
\hline W-box & -898 & -663 & -428 & -192 & & & Wounding and GA response element \\
\hline ERE & -1429 & & & & & & Ethylene-responsive element \\
\hline Blue or UV-B & -1350 & -414 & -801 & & & & Blue and UV-B response \\
\hline
\end{tabular}

\section{Construction of expression vector}

The full-length sequences of CHSP (CHSP1) and five other segments (CHSP2, CHSP3, CHSP4, CHSP5 and CHSP6) were linked to pBI121, with the 35S CaMV promoter removed using PCR to obtain pBI121+CHSP1 CHSP6, to evaluate the reliability of the upstream encoding sequence of the $C H S$ gene in regulating gene expression precisely.

Sequencing revealed that CHSP was positively inserted into the upstream region of the GUS gene and had the same size as the design. pBI121 without the $35 \mathrm{~S}$ promoter and the positive plant transformed from $\mathrm{pBI} 121$ were taken as the negative control and positive control, respectively (Figure 2).

\section{CHSP promoter is systemically responsive to nonlethal stress or plant growth regulators}

To define more precisely the pattern of expression driven by the promoter region, we generated transgenic $N$. tabacum plants expressing the GUS gene under the control of the 2273 bp 5' region of the CHS promoter. Using Southern-blot analysis in the T0 generation, single copy insert lines were selected for further study.

QRT-PCR analysis was adopted to examine whether ultraviolet rays, hormones, and injury 
treatment could change the expression pattern of GUS driven by CHSP in transgenic tobacco (Table 3). Previous studies showed that $100 \mathrm{mg} / \mathrm{L} \mathrm{ABA}$ could improve the enzymatic activity of Ginkgo PAL, CHS, and CHI (Xu et al., 2007a, 2008; Cheng et al., 2011). The present study used the same concentration of ABA to treat the transgenic tobacco leaves. The transcription level of GUS driven by CHSP was stable during the first $2 \mathrm{~h}$, increased rapidly at $4 \mathrm{~h}$ (sampling point, $\sim 1.8$ times the control), and then decreased slowly (Figure 3A). Cu ions played a crucial role in inducing GUS. The transcription level increased rapidly during the first $12 \mathrm{~h}$ of spraying $(\sim 7.13$ times the control level at $12 \mathrm{~h})$ and decreased $12 \mathrm{~h}$ after (Figure 3B). Under low-dose UV-B treatment, the transcription level of GUS increased slowly at first and then decreased rapidly, reaching maximum expression after approximately $12 \mathrm{~h}$ ( $\sim 2.8$ times the control level) (Figure 3C). In contrast to ABA treatment, the expression level of GUS in ETH spraying increased rapidly and steadily and lasted long. It reached the maximum level (3.1 times the reference level) at $8 \mathrm{~h}$, after which a high transcription level was maintained. It declined rapidly to the reference level after approximately $16 \mathrm{~h}$ (Figure 3D). As to injury treatment (WOU), the transcription level fluctuated, dropped slightly after $2 \mathrm{~h}$ (sampling point), rose slowly after $4 \mathrm{~h}$, reached the maximum level at $12 \mathrm{~h}$ (sampling point, $\sim 2.4$ times the control level), and then started to decline (Figure 3E). Contrarily, GA treatment suppressed the transcription of GUS by CHSP: the transcription level of GUS in tobacco leaves increased slightly at $2 \mathrm{~h}$ (but responded slowly in contrast to other hormones) and then decreased rapidly. It reached the minimum level after approximately $8 \mathrm{~h}$ ( $\sim .6$ times the reference level) and then increased slowly (Figure 3F). Of all types of treatment, only GA reduced the transcription level of GUS.

\begin{tabular}{|c|c|c|c|}
\hline \multirow[t]{2}{*}{ Treatment } & \multirow[t]{2}{*}{ Time (hours) } & \multicolumn{2}{|c|}{ Relative expression level } \\
\hline & & $\mathrm{CK}$ & Sample \\
\hline \multirow[t]{5}{*}{$\mathrm{ABA}$} & 4 & $5.36 \pm 0.39$ & $5.45 \pm 0.26$ \\
\hline & 8 & $4.89 \pm 0.31$ & $8.94 \pm 1.26$ \\
\hline & 12 & $5.13 \pm 1.10$ & $6.07 \pm 0.81$ \\
\hline & 24 & $5.13 \pm 0.78$ & $5.88 \pm 0.60$ \\
\hline & 48 & $4.89 \pm 0.52$ & $5.26 \pm 0.42$ \\
\hline \multirow[t]{5}{*}{$\mathrm{Cu}$} & 4 & $5.36 \pm 0.33$ & $7.97 \pm 0.66$ \\
\hline & 8 & $5.44 \pm 0.87$ & $11.34 \pm 1.64$ \\
\hline & 12 & $5.46 \pm 0.77$ & $22.38 \pm 1.49$ \\
\hline & 24 & $5.57 \pm 0.45$ & $31.89 \pm 2.08$ \\
\hline & 48 & $5.45 \pm 0.53$ & $21.75 \pm 1.31$ \\
\hline \multirow[t]{5}{*}{ UV-B } & $\begin{array}{r}40 \\
4\end{array}$ & $5.25 \pm 0.97$ & $\begin{array}{r}4.77 \pm 0.43 \\
\end{array}$ \\
\hline & 8 & $5.44 \pm 0.71$ & $7.57 \pm 0.89$ \\
\hline & 12 & $5.13 \pm 0.42$ & $13.88 \pm 1.28$ \\
\hline & 24 & $5.02 \pm 0.75$ & $14.78 \pm 1.03$ \\
\hline & 48 & $4.56 \pm 0.77$ & $6.64 \pm 0.76$ \\
\hline \multirow[t]{5}{*}{ ETH } & 4 & $5.14 \pm 1.16$ & $4.43 \pm 0.64$ \\
\hline & 8 & $5.11 \pm 0.67$ & $13.46 \pm 2.19$ \\
\hline & 12 & $5.35 \pm 0.52$ & $18.10 \pm 1.52$ \\
\hline & 24 & $4.91 \pm 0.68$ & $17.00 \pm 2.67$ \\
\hline & 48 & $4.78 \pm 0.79$ & $6.86 \pm 0.58$ \\
\hline \multirow[t]{5}{*}{ WOU } & 4 & $5.58 \pm 0.37$ & $4.13 \pm 0.59$ \\
\hline & 8 & $5.22 \pm 0.46$ & $7.08 \pm 1.32$ \\
\hline & 12 & $5.68 \pm 1.18$ & $9.55 \pm 1.36$ \\
\hline & 24 & $5.57 \pm 0.54$ & $14.71 \pm 1.69$ \\
\hline & 48 & $4.45 \pm 0.78$ & $8.73 \pm 0.42$ \\
\hline \multirow[t]{5}{*}{ GA } & 4 & $5.16 \pm 0.54$ & $5.66 \pm 0.80$ \\
\hline & 8 & $5.15 \pm 0.39$ & $4.29 \pm 0.51$ \\
\hline & 12 & $4.41 \pm 0.48$ & $3.34 \pm 0.44$ \\
\hline & 24 & $5.57 \pm 0.46$ & $3.68 \pm 0.51$ \\
\hline & 48 & $5.07 \pm 0.47$ & $5.45 \pm 0.85$ \\
\hline
\end{tabular}

Data are reported as means $\pm \mathrm{SD}(\mathrm{N}=3)$. $\mathrm{ABA}=$ abscisic acid; $\mathrm{Cu}=$ copper; $\mathrm{ETH}=$ ethephon; $\mathrm{WOU}=$ wounding; $\mathrm{GA}=$ gibberellin. 


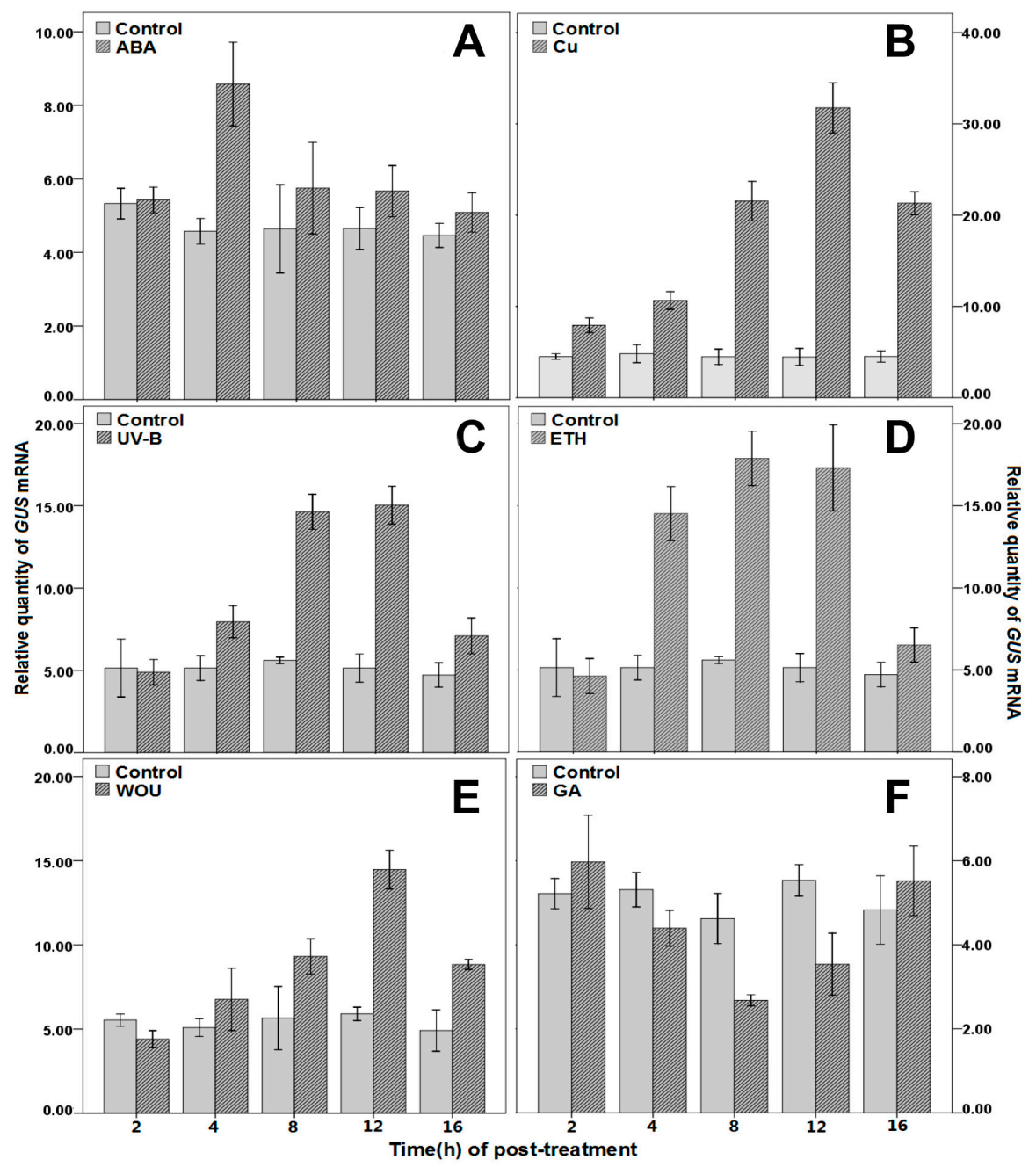

Figure 3. Quantitative analyses of GUS transcription in transgenic CHSP-GUS plants in response to A. ABA, B. $\mathrm{Cu}$, C. UV-B, D. ETH, E. WOU, F. GA. Relative quantities of GUS mRNA at various time points post-treatment with each sample was individually assayed in triplicate. Values shown represent the mean reading from three treated plants and the error bars indicated the standard errors of the mean.

\section{Deletion analysis of the CHSP promoter in transgenic tobacco}

To localize the promoter region involved in the control of the expression of CHS in relation to the cell cycle, sequential deletions were introduced in the sequence upstream of the GUS reporter gene. GUS activity was measured in proteins extracted from tobacco cells stably transformed with the different constructs. Several independent transgenic lines were analyzed for each construct. Proteins were extracted from two-week-old seedlings, and the results are presented in Table 4. In a fortnight-old culture (Figure 4), the maximum activity and expression levels were 
obtained with the complete promoter (CHSP1). A deletion of $331 \mathrm{bp}$ at its $5^{\prime}$ end (CHSP2) induced a significant decrease in GUS activity of about $12.1 \%$ and in expression level of about $20.67 \%$. Removal of the next 348 bp resulted in less variation of GUS activity (CHSP3), with a decrease in GUS activity of about $9.1 \%$ and increase in expression level of about $1.1 \%$. GUS activity dropped dramatically with the CHSP4 constructs (about $36 \%$, a deletion of 376 bp at its $5^{\prime}$ end). Similar results were measured for GUS expression level. A deletion of the next 508 bp upstream did not result in a significant variation in GUS expression (CHSP5), but there was a sharp decline in activity. However, a further deletion containing only $306 \mathrm{bp}$ upstream of the transcription start site, but still the two TATA boxes and the CCAAT box, almost completely abolished GUS expression and activity (CHSP6). GUS activity dropped dramatically with the CHSP4 constructs and was almost totally absent with the CHSP6 construct (Table 4). An intended observation was that the CHSP1 complete promoter conferred a similar GUS expression and activity as the $35 \mathrm{~s}$ CaMS promoter. In other respects, in wild-type NC89, the level of GUS activity was similar to that obtained with proteins extracted from $\mathrm{pBI} 121-35 \mathrm{~S}$ transgenic cells.

Table 4. GUS activity and the relative expression of GUS mRNA driven by dfferent promoter sequence.

\begin{tabular}{lcr}
\hline Different vectors & Relative expression level & GUS activity \\
\hline CHSP1 & $32.39 \pm 0.83$ & $12.5131 \pm 0.3309$ \\
CHSP2 & $26.93 \pm 0.95$ & $11.1874 \pm 0.1905$ \\
CHSP3 & $27.96 \pm 0.29$ & $10.0580 \pm 0.2168$ \\
CHSP4 & $15.37 \pm 0.33$ & $6.1880 \pm 0.4533$ \\
CHSP5 & $14.86 \pm 0.19$ & $2.8248 \pm 0.3989$ \\
CHSP6 & $3.92 \pm 0.34$ & $1.9592 \pm 0.2610$ \\
pBI121 & $32.15 \pm 0.38$ & $12.4893 \pm 0.2559$ \\
pBI121-35S & $0.06 \pm 0.07$ & $0.0011 \pm 0.0001$ \\
\hline
\end{tabular}

Data are reported as means $\pm \mathrm{SE}$ of triplicate tests.

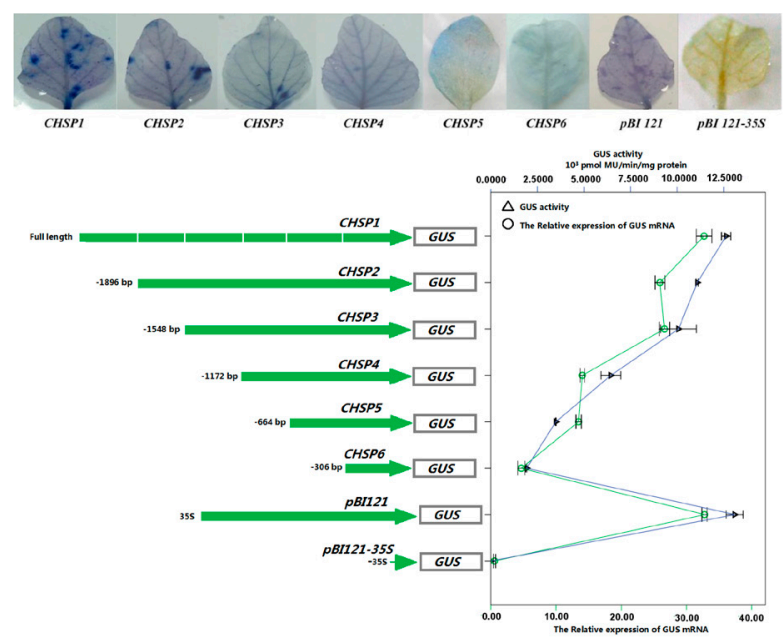

Figure 4. GUS activity and the relative expression of GUS mRNA. Schematic diagram of the constructs used for GUS activity assays in leaves of transgenic tobacco plants are shown at left. The plasmid pBI121 containing a promoterless GUS gene was used as negative control. Quantitative analyses of GUS transcription and activity of transgenic plants driven by deletion constructs of CHSP promoter are shown at right. Error bars represent standard deviation $(\mathrm{SD})$. Data are reported as means $\pm \mathrm{SD}$ of triplicate tests. 
Two conclusions could be drawn from these results obtained in transgenic tobacco. On the one hand, the CHS promoter belongs to a flavonoid synthesis gene, which is usually induced by environment and hormone. In a normal growth condition, especially in tobacco, the $35 \mathrm{~S}$ promoter strongly enhances the level of expression of the gene fusion. On the other hand, the proximal promoter region up to $-306 \mathrm{bp}$ is not sufficient to drive a significant expression of the chimeric construct.

\section{DISCUSSION}

The 2273-bp promoter sequence upstream of the CHS gene's transcription start site was cloned using Genomic Walking technology and named "GbCHSP". Bioinformatics analysis was carried out on the regulated region. The expression carrier pBI121+CHSP $1 \sim \mathrm{CHSP} 6$ and the control carrier pBI121-35S were built and transcribed to tobacco for primary verification of their functions and to further examine the function of the CHSP regulatory region.

Endogenous hormones have been shown to be associated with changes in flavone content during the synthesis of Ginkgo flavonoids. On the other hand, ethylene and ABA promote the synthesis of flavonoids. Increased concentrations of ethylene and ABA benefit the accumulation of Ginkgo flavones (Wang et al., 2002; Cheng et al., 2004), and treatment of transgenic tobacco with ethylene and ABA could enhance transcription levels of the GUS gene through GbCHSP. This promoter contains ERE and DPBF elements, indicating that the role of ethylene and $\mathrm{ABA}$ in promoting the accumulation of Ginkgo flavones is associated with the enhanced expression of $C H S$ as induced by ERE and DPBF elements in GbCHSP. Moreover, CHSP contains the acting element W-box unit with five WRKT factors, and the transcription level of WRKY is suppressed by GA (Zhang et al., 2004). Based on these data, high concentrations of exogenous GA suppress the expression of WRKY and further suppress the transcriptional regulation of GbCHSP. In addition, the transcription level of GUS in tobacco is suppressed by GA. Earlier research has shown that high levels of endogenous GA in plants do not help synthesize flavonoids (Hinderer et al., 1984) and the accumulation of Ginkgo flavones is also suppressed by endogenous GA (Cheng et al., 2004). As such, GA's suppression of Ginkgo flavone accumulation is associated with reduced expression of the CHS gene. Exogenous CTKs reportedly help improve the expression level of the CHS gene in $A$. thaliana (Deikman and Hammer, 1995). The changing endogenous CTKs/ABA ratio inside Ginkgo leaves affects the accumulation of flavones (Cheng et al., 2004). GbCHSP contains the CPB unit, which binds to CTKs and proteins, indicating that changing levels of Ginkgo CHS expression are associated with the interaction between CTKs and other hormones. The ARF factor aggregate unit and TGA-box found in CHSP are involved in the regulation of gene expression by auxin, which binds to the ARF protein and then acts on the transcription of positive regulatory genes in the promoter region (Liu et al., 1994). Auxin is thus expected to help improve the transcription level of Ginkgo CHS. However, whether it benefits the accumulation of flavones has yet to be studied.

Accumulation of flavone is also affected by external factors, as reflected by $\mathrm{GbCH}$ $S P$ analysis. CHSP scanning has shown a number of binding sites of UV-B response factors similar to those in A. thaliana. In Ginkgo, the expression level of CHS gene is increased by ultraviolet rays (Pang et al., 2005), which is closely related to the ultraviolet response unit in GbCHSP and is confirmed by increased transcription levels of GUS induced by UV-B by 
GbCHSP. Ginkgo CHSP contains a number of Ca response element, and such units contain ABRE-like motifs. An ABRE tetramer bound to a Ca response element initiates signal transduction associated with changing concentrations of $\mathrm{Ca}^{2+}$ ions in the cytoplasm. Furthermore, $\mathrm{Ca}^{2+}$ mediates a number of signal transduction events (Kaplan et al., 2006). Hence, environmental factors likely exert their effects on $C H S$ expression via the ABRE unit in CHSP. Low concentrations of $\mathrm{Cu}^{2+}$ provide flavone to potted seedlings of Ginkgo and prolong the best harvest period (Wang et al., 2007). GbCHSP also contains a number of CuRE binding sequences, and this element is subject to positive induction (transcribed to protein) by $\mathrm{Cu}^{2+}$ signals (Quinn et al., 2000). These data suggest that increased transcription levels of GUS in transgenic tobacco and accumulation of Ginkgo flavone caused by $\mathrm{Cu}^{2+}$ are associated with elevated transcription levels of GbCHS as regulated by CuRE. When tobacco is injured by external factors, the WRKY factor could bind rapidly to the W-box of the acting element and induce the expression of relevant genes (Nishiuchi et al., 2004). Injury treatment of transgenic tobacco has also proven that external mechanical injury could induce GUS expression through GbCHSP. GbCHSP contains three apparent W-boxes that provide the molecular basis for the enhanced expression of $C H S$ and flavone content under the injury stimulus (Pang et al., 2005). Moreover, a number of GT elements at the upstream region of GbCHSP may play a role in expression induced by pathogenic bacteria and salt stress (Park et al., 2004), but evidence to support this currently does not exist.

For an in-depth analysis of the functional regulatory region of GbCHSP, the promoter was divided into five segments from the $5^{\prime}$ end and each segment's induction mechanism for the transcription of the GUS gene was examined. The results indicated that pruning at the $5^{\prime}$ upstream sequence yields a rapid decline in GUS gene transcription and enzymatic activity. Particularly, pruning of sequences enriched with regulatory elements has greater effects. Similar results have been reported by other studies. A transgenic study of the fusion carrier of the Capsicum annuum cakn promoter and GUS found that a complete cakn promoter sequence could significantly raise the transcription level of GUS genes to levels higher than those of the $35 \mathrm{~S}$ promoter carried by the carrier itself (Schantz et al., 2005). Subsequently pruned segments of promoters have been shown to reduce the transcription level of GUS gradually. CAAT-box and TATA-box regulatory units by themselves are not sufficient enough to drive high-level GUS gene transcription (Schantz et al., 2005). Research into Populus trichocarpa has demonstrated that, as the promoter segments are shortened, especially with less W-box regulatory units, injury treatment increasingly weakens the induction of PtrCHS4 on the reporter gene (Sun et al., 2011). Sequence analysis has found only one Inr element between 327 and $680 \mathrm{bp}$ (CHSP2, CHSP3). Inr elements shoulder some functions of transcriptional activation (particularly, response to photoinduction) in promoter sequences that lack the TATA-box (Nakamura et al., 2002). Therefore, in CHSP rich in TATA box elements, pruning this sequence will not have apparent effects on the transcription level. Analysis of promoter segments determined that the pruned CHSP3 and CHSP4 segments have particularly remarkable effects on the transcription level and enzymatic activity of GUS. One Q-element unit between CHSP3 and CHSP4, a transcription-enhancing signal in the gene promoter of maize ZM13 (Hamilton et al., 1998) is possibly the main cause of the rapid decline in the transcription regulatory function of the CHSP4 segment. Another site of CHSP (between -664 to -306, CHSP5 CHSP6) exhibited rapid reduction, which contains a large amount of acting elements, such as EEC, W box, GATA motif, CAAT box, and TATA box. Among others, CAAT box and TATA box are 
crucial in regulating transcription enhancement, and the GATA motif helps regulate photoinduction. Hence, the absence of these key elements is likely responsible for the reduced ability of GbCHSP to regulate transcription in tobacco leaves. GbCHSP6 contains the basic transcription regulatory units CAAT box and TATA box; CIR, GC-motif, STK-1, and W box are among those acting elements that could also provide weak transcription signals (Sun et al., 2011). These data suggest that the short CHSP6 could also regulate weak transcription of GUS genes.

The CHS gene is essential to the metabolism of Ginkgo flavonoids (Cheng et al., 2009). The key to increasing the flavonoid content of leaves of a Ginkgo plant is to improve the expression level of key genes in specific tissues during the developmental period. Cloning the GbCHSP of Ginkgo and studying its mechanisms of action will add substantially to the current knowledge of the regulation of GbCHS gene expression.

\section{ACKNOWLEDGMENTS}

Research supported by Economic Forest Germplasm Improvement and Comprehensive Utilization of Resources of Hubei Key Laboratories (\#20011BLKF238, \#2013000203 and \#20011BLKF240, the Natural Science Foundation of China (\#30971974 and \#31270717), and the University Industry Cooperation Fund of Hubei Educational Office (\#CXY2009B009).

\section{REFERENCES}

Cheng S, Wang Y, Li J, Fei Y, et al. (2004). Study on the relationship between the endogenous hormones and flavonoids in Ginkgo biloba leaf. Sci. Silvae Sin. 40: 45-49.

Cheng SY, Xu F and Wang Y (2009). Advances in the study of flavonoids in Ginkgo biloba leaves. J. Med. Plants Res. 3: $1248-1252$.

Cheng H, Li L, Cheng S, Cao F, et al. (2011). Molecular cloning and function assay of a chalcone isomerase gene (GbCHI) from Ginkgo biloba. Plant Cell Rep. 30: 49-62.

da Costa e Silva, Klein L, Schmelzer E, Trezzini GF, et al. (1993). BPF-1, a pathogen-induced DNA-binding protein involved in the plant defense response. Plant J. 4: 125-135.

Deikman J and Hammer PE (1995). Induction of anthocyanin accumulation by cytokinins in Arabidopsis thaliana. Plant Physiol. 108: 47-57.

Hamilton DA, Schwarz YH and Mascarenhas JP (1998). A monocot pollen-specific promoter contains separable pollenspecific and quantitative elements. Plant Mol. Biol. 38: 663-669.

Hartmann U, Valentine WJ, Christie JM, Hays J, et al. (1998). Identification of UV/blue light-response elements in the Arabidopsis thaliana chalcone synthase promoter using a homologous protoplast transient expression system. Plant Mol. Biol. 36: 741-754.

Heller W and Hahlbrock K (1980). Highly purified "flavanone synthase" from parsley catalyzes the formation of naringenin chalcone. Arch. Biochem. Biophys. 200: 617-619.

Hinderer W, Petersen M and Seitz HU (1984). Inhibition of flavonoid biosynthesis by gibberellic acid in cell suspension cultures of Daucus carota L. Planta 160: 544-549.

Hirsinger C, Salva I, Marbach J, Durr A, et al. (1999). The tobacco extensin gene Ext 1.4 is expressed in cells submitted to mechanical constraints and in cells proliferating under hormone control. J. Exp. Bot. 50: 343-355.

Jefferson RA, Kavanagh TA and Bevan MW (1987). GUS fusions: beta-glucuronidase as a sensitive and versatile gene fusion marker in higher plants. EMBO J. 6: 3901-3907.

Jiang L and Cai LH (2000). A method for extracting DNA of Ginkgo biloba. Plant Physiol. Com. 36: 340-341.

Kaplan B, Davydov O, Knight H, Galon Y, et al. (2006). Rapid transcriptome changes induced by cytosolic $\mathrm{Ca}^{2+}$ transients reveal ABRE-related sequences as $\mathrm{Ca}^{2+}$-responsive cis elements in Arabidopsis. Plant Cell 18: 2733-2748.

Kiba A, Toyoda K, Ichinose Y, Yamada T, et al. (1995). Specific inhibition of cell wall-bound ATPases by fungal suppressor from Mycosphaerella pinodes. Plant Cell Physiol. 36: 809-817.

Koes R, Verweij W and Quattrocchio F (2005). Flavonoids: a colorful model for the regulation and evolution of biochemical pathways. Trends Plant Sci. 10: 236-242. 
Li LL, Cheng H, Peng JY and Cheng SY (2010). Construction of a plant expression vector of chalcone synthase gene of Ginkgo biloba and its genetic transformation into tobacco. Front. Agric. China 4: 456-462.

Liu Y, Lou Q, Xu W, Xin Y, et al. (2011). Characterization of a chalcone synthase (CHS) flower-specific promoter from Lilium orential 'Sorbonne'. Plant Cell Rep. 30: 2187-2194.

Liu ZB, Ulmasov T, Shi X, Hagen G, et al. (1994). Soybean GH3 promoter contains multiple auxin-inducible elements. Plant Cell 6: 645-657.

Nakamura M, Tsunoda T and Obokata J (2002). Photosynthesis nuclear genes generally lack TATA-boxes: a tobacco photosystem I gene responds to light through an initiator. Plant J. 29: 1-10.

Nakatsuka A, Izumi Y and Yamagishi M (2003). Spatial and temporal expression of chalcone synthase and dihydroflavonol 4-reductase genes in the Asiatic hybrid lily. Plant Sci. 165: 759-767.

Nishiuchi T, Shinshi H and Suzuki K (2004). Rapid and transient activation of transcription of the ERF3 gene by wounding in tobacco leaves: possible involvement of NtWRKYs and autorepression. J. Biol. Chem. 279: 55355-55361.

Pang Y, Shen G, Wu W, Liu X, et al. (2005). Characterization and expression of chalcone synthase gene from Ginkgo biloba. Plant Sci. 168: 1525-1531.

Park HC, Kim ML, Kang YH, Jeon JM, et al. (2004). Pathogen- and NaCl-induced expression of the SCaM-4 promoter is mediated in part by a GT-1 box that interacts with a GT-1-like transcription factor. Plant Physiol. 135: 2150-2161.

Porebski S, Bailey LG and Baum BR (1997). Modification of a CTAB DNA extraction protocol for plants containing high polysaccharide and polyphenol components. Plant Mol. Biol. Rep. 15: 8-15.

Quinn JM, Barraco P, Eriksson M and Merchant S (2000). Coordinate copper- and oxygen-responsive Cyc6 and Cpx1 expression in Chlamydomonas is mediated by the same element. J. Biol. Chem. 275: 6080-6089.

Schantz ML, Jamet E, Guitton AE, Schantz R, et al. (2005). Functional analysis of the bell pepper KNOLLE gene (cakn) promoter region in tobacco plants and in synchronized BY2 cells. Plant Sci. 169: 155-163.

Schmid J, Doerner PW, Clouse SD, Dixon RA, et al. (1990). Developmental and environmental regulation of a bean chalcone synthase promoter in transgenic tobacco. Plant Cell 2: 619-631.

Smith JV and Luo Y (2004). Studies on molecular mechanisms of Ginkgo biloba extract. Appl. Microbiol. Biotechnol. 64: 465-472.

Sun Y, Tian Q, Yuan L, Jiang Y, et al. (2011). Isolation and promoter analysis of a chalcone synthase gene PtrCHS4 from Populus trichocarpa. Plant Cell Rep. 30: 1661-1671.

van Beek TA (2002). Chemical analysis of Ginkgo biloba leaves and extracts. J. Chromatogr. A 967: 21-55.

van der Meer IM, Brouwer M, Spelt CE, Mol JN, et al. (1992). The TACPyAT repeats in the chalcone synthase promoter of Petunia hybrida act as a dominant negative cis-acting module in the control of organ-specific expression. Plant $J$. 2: 525-535.

Wang Y, Cheng S, Fei Y, Li J, et al. (2002). Studies on the effects of regulating measures on the flavonoids contents in Ginkgo biloba leaves. Hubei Agric. Sci. 40: 103-105.

Wang Y, Li L, Xu F and Cheng S (2007). Effects of some metal ions on phenylalanine ammonia-lyase activities and flavonoids content of Ginkgo biloba leaves in the potted orchard. J. Nanjing Forest Univ. (Nat. Sci. Edit.) 31: 68-72.

Weisshaar B, Armstrong GA, Block A, da Costa e Silva, et al. (1991). Light-inducible and constitutively expressed DNAbinding proteins recognizing a plant promoter element with functional relevance in light responsiveness. EMBO J. 10: $1777-1786$.

Xu F (2005). Molecular Cloning and Expression of Chalcone Synthase Gene and Phenylalanine Ammonia-Lyase Gene from Ginkgo biloba. Master's thesis, Huazhong Agricultural University, Wuhan.

Xu F, Cheng SY, Cheng SH, Wang Y, et al. (2007a). Time course of expression of chalcone synthase gene in Ginkgo biloba. Zhi. Wu Sheng Li Yu Fen. Zi. Sheng Wu Xue Xue Bao 33: 309-317.

Xu F, Cheng S, Wang Y, Li L, et al. (2007b). Efficient amplification and sequence analysis of chalcone synthase gene from Ginkgo biloba by thermal asymmetric interlaced PCR. J. Fruit Sci. 24: 237-243.

Xu F, Cai R, Cheng S, Du H, et al. (2008). Molecular cloning, characterization and expression of phenylalanine ammonialyase gene from Ginkgo biloba. Afr. J. Biotechnol. 7: 721-729.

Yu LM, Lamb CJ and Dixon RA (1993). Purification and biochemical characterization of proteins which bind to the H-box cis-element implicated in transcriptional activation of plant defense genes. Plant J. 3: 805-816.

Zhang ZL, Xie Z, Zou X, Casaretto J, et al. (2004). A rice WRKY gene encodes a transcriptional repressor of the gibberellin signaling pathway in aleurone cells. Plant Physiol. 134: 1500-1513. 